\title{
Performance of muon and tau identification at ATLAS
}

\author{
Mansoora Shamim ${ }^{* \dagger}$ \\ On behalf of the ATLAS Collaboration \\ University of Oregon \\ E-mail: mansoora.shamimecern.ch
}

Charged leptons play an important role in the physics programme at the Large Hadron Collider. The performance of reconstructions and identification of charged leptons, therefore, must be known with high precision. In 2012 the LHC is operated in a mode leading to up to 40 inelastic pp collisions per bunch crossing, so-called "pile-up", with an average number of 20 inelastic collisions. This contribution presents performance of the muon and tau reconstructions and identification in the high pile-up environment at the ATLAS experiment.

36th International Conference on High Energy Physics

4-11 July 2012

Melbourne, Australia

\footnotetext{
* Speaker.

${ }^{\dagger}$ Eric Torrence presented slides at ICHEP.
} 


\section{Introduction}

Muons and taus play an important role in the physics programme at the Large Hadron Collider. In this contribution, di-muon decays of $J / \psi$ mesons and $\mathrm{Z}$ bosons have been used to study the muon reconstruction and identification efficiency as a function of the muon transverse momentum and the number of inelastic collisions per event. Optimal identification of hadronically decaying taus is achieved by using detailed information from tracking and calorimeter detector components. The identification efficiencies are measured using $W \rightarrow \tau \nu$ and $Z \rightarrow \tau \tau$ events. The energy scale uncertainties for taus are determined by investigating the single hadron calorimeter response etc., as well as kinematic distributions in $Z \rightarrow \tau \tau$ events.

\section{ATLAS detector}

The ATLAS detector [1] is a seven story high machine with many subdetectors layered in an onion-like structure. At its very core is a high precision tracking system for charged particles in a solenoidal magnetic field, which does momentum measurement and vertex reconstruction. It can also measure transition radiation to distinguish electrons and charged pions. Surrounding this tracking system are the electromagnetic and hadronic calorimeters, dedicated to stopping all electrons, photons and hadrons and to measuring their energy. The only detectable particles not stopped by the calorimeters are muons, which are what the outermost system is dedicated to. The muon system is capable of tracking and triggering on muons. It has its own magnetic field, provided by three gigantic air-core toroids.

\section{Muon reconstruction and identification}

\subsection{Muon reconstruction}

The ATLAS muon system is optimized for muon identification, with an efficiency greater than 95\% and a relative momentum resolution better than $3 \%$ over a wide transverse momentum $\left(p_{T}\right)$ range and $10 \%$ at $p_{T}=1 \mathrm{TeV}$. The Inner Detector (ID) and the Muon Spectrometer (MS) provide independent measurement of the muon momentum. The hit inofrmation in three layers of precision drift tubes (MDT) chambers in $|\eta|<2$ and two layers of the MDT chambers in combination with one layer of cathode strip chambers (CSC) at the entrance of the MS for $2 \leq|\eta| \leq 2.7$ is also used in the reconstruction of muons. The resistive-plate chambers (RPC) in the barrel region and the thin-gap chambers (TGC) in the end-caps provide triggering capabilities and also measure the muon trajectory in the non-bending plane of the spectrometer magnets.

\subsection{Muon reconstruction and identification strategies}

In ATLAS, four kinds of muon candidates are distinguished depending on the way they are reconstructed:

Stand-alone muon: The spectrometer track is entirely reconstructed in the MS and then extrapolated back to the beam line to determine the direction of flight and the impact parameter of the muon at the interaction point. To obtain the muon momentum at the interaction point, the muon momentum measured in the MS is corrected for the parametrized energy loss in the calorimeter. 


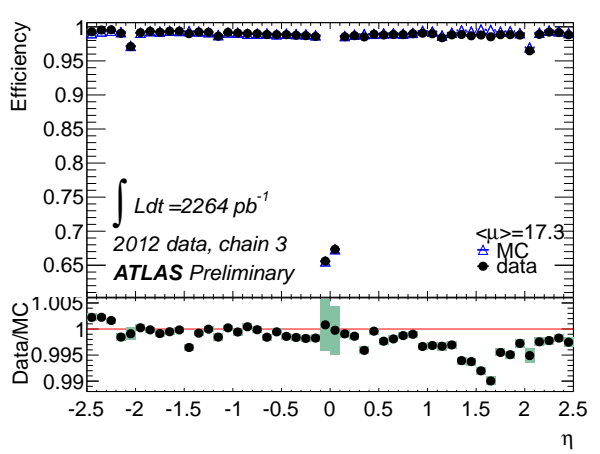

Figure 1: Reconstruction efficiency for combined and segment tagged muons versus the muon pseudorapidity [4]. Green bands represent the statistical uncertainty.

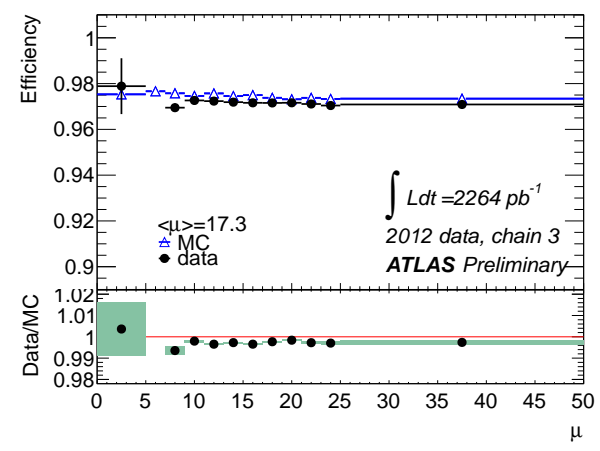

Figure 2: Reconstruction efficiency for combined and segment tagged muons versus the average number of inelastic pp collisions per bunch crossing [4]. Green bands represent the statistical uncertainty.

Combined muon: Tracks result from the combination of MS and ID measurements by a statistical combination or a refit of the entire track. Energy losses in the calorimeter are taken into account using parametrization and possibly calorimeter measurements. This reconstruction strategy provides the most precise measurement of the momentum and position of a muon.

Segment tagged muon: If a track in the ID, extrapolated to the MS, is associated with straight track segments in the precision muon chambers, it is identified as a muon. This provides efficiency recovery in regions with low MS detector coverage.

Calorimeter tagged muon: An ID track with an energy deposition in the calorimeters compatible with a minimum ionizing particle is identified as a muon.

\subsection{Muon reconstruction efficiency}

The muon reconstruction efficiency is measured as a function of $\eta$ and of, $\mu$ which represents the average number of inelastic pp collisions in a given bunch crossing [2, 3]. The muon reconstruction efficiencies measured with experimental data using muon pairs produced in the decays of $\mathrm{Z}$ bosons are compared with those predicted by the simulation. In this study, the tag-and-probe method is used: $Z \rightarrow \mu \mu$ events with one well reconstructed muon, the tag, and one oppositely charged track measured by the ID, the probe, are selected. To reduce the backgrounds, the invariant mass of the pair has to be close to the nominal $\mathrm{Z}$ mass to match the tag together with the probe to the signature of a $\mathrm{Z}$ boson decay. The reconstruction efficiency is determined from the fraction of probes which are matched to a reconstructed muon. The muon reconstruction efficiency for combined and segment tagged muons, vs $\eta$ and $\mu$ are shown in Figs. 1 and 2. In Fig. 2 one can see that increasing pile-up has no significant effect on the efficiency. Furthermore, the muon reconstruction efficiency is above $98 \%$ in most of the $\eta$ range and data to simulation ratio lies within $1 \%$.

\subsection{Muon momentum resolution}

A measurement of the muon momentum resolution is performed using $824 \mathrm{pb}^{-1}$ of data collected in early 2012 and compared to a $Z \rightarrow \mu \mu$ simulation sample [5]. The di-muon invariant mass 


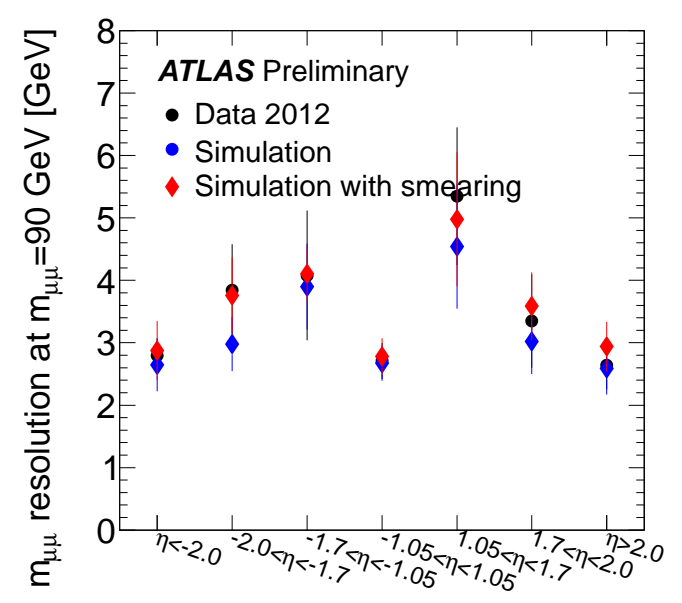

Figure 3: Di-muon mass resolution for muons from $\mathrm{Z}$ boson decays using the momentum measurements in the muon spectrometer corrected for the energy loss in the calorimeters [4].

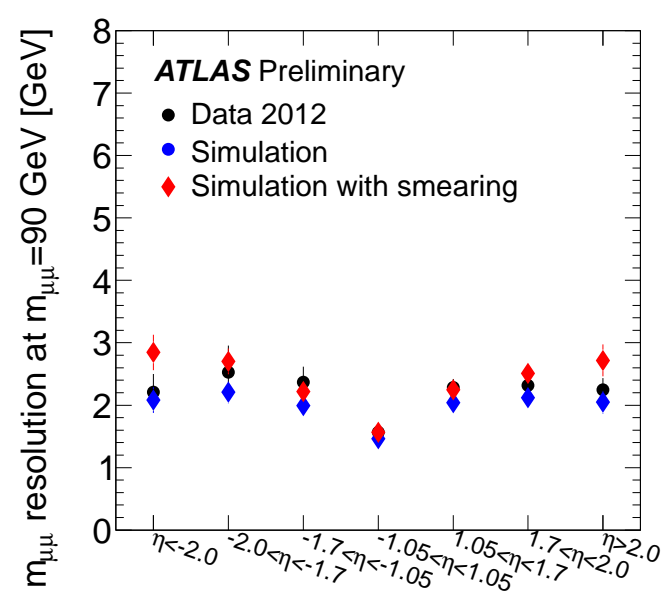

Figure 4: Di-muon mass resolution for muons from $\mathrm{Z}$ boson decays using the combined muon momentum measurements performed in the inner detector and the muon spectrometer [4].

distributions are obtained separately from MS and ID track parameters and integrated over all muon $p_{T}$ values. The resolution is the width of the Gaussian which is convoluted with the Breit-Wigner shape in $Z \rightarrow \mu \mu$ decays at generator level. The fit is performed in the mass window $m_{\mu \mu} \in[75$, 105] GeV. Figures 3 and 4 show the mass resolutions for different $\eta$ regions as measured in the MS only, and combined MS and ID, respectively. Error bars are the sum of the statistical error and the absolute value of the change of the resolution when the fit range is reduced to $m_{\mu \mu} \in[82,100]$ $\mathrm{GeV}$ from $m_{\mu \mu} \in[75,105] \mathrm{GeV}$. The results obtained are are from first pass reconstruction of 2012 data, done just after data taking. It is expected that after data reprocessing with new alignment the resolution will improve. The resolution of the muons is underestimated in the simulation due to inaccuracies in the modeling of the alignment for the ID and MS. Consequently the muon momenta in simulation are smeared and rescaled to correct the central value and apply uncertainties. Figures 3 and 4 show that after applying the smearing to simulation, the measurements agree better with the observed values in data.

\section{Tau reconstruction and identification}

\subsection{Reconstruction}

The majority of hadronic tau decays are characterized by one or three charged pions accompanied by neutral pions. In ATLAS tau reconstruction and identification [6] concentrates on the hadronic decay modes of a tau. They are classified according to the number of charged decay particles (also called 'prongs' in this text). These decays can be differentiated from QCD jets by their characteristics, such as low track multiplicity, collimated energy deposits, and in case of 3-prong taus the displacement of the secondary vertex.

Calorimeter jets with a transverse energy larger than $10 \mathrm{GeV}$ and within the detector acceptance are used as a seed for the reconstruction of tau candidates. Tracks within a cone of 


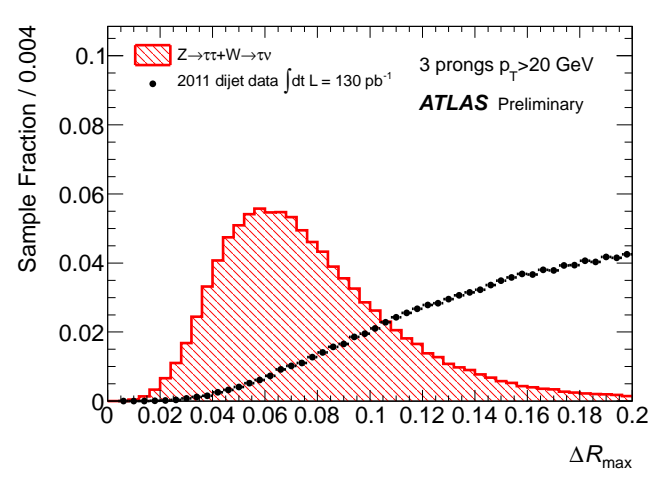

Figure 5: Maximal distance between a track and the tau axis, $\Delta \mathrm{R}_{\max }$. Only tracks inside a cone of $\Delta R<0.2$ around the tau axis are considered [6].

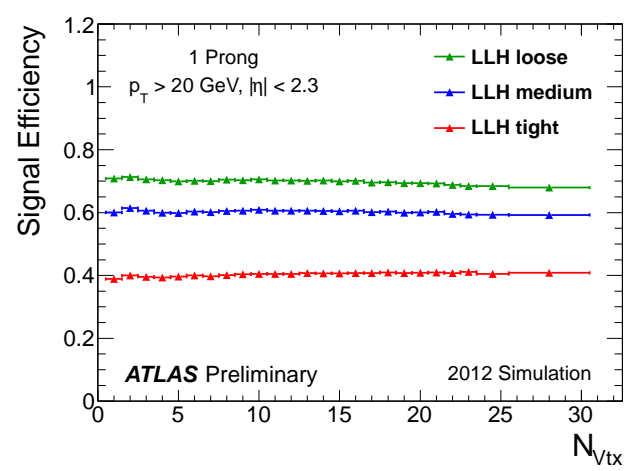

Figure 6: Signal efficiencies for 1-prong $\tau$ candidates as a function of the number of reconstructed vertices [7].

$\Delta \mathrm{R}=\sqrt{(\Delta \phi)^{2}+(\Delta \eta)^{2}}<0.4$ around the tau axis passing certain quality criteria are associated to the tau candidate and used to calculate the discriminating variables. The tau energy is calculated using all calorimeter clusters within a core of $\Delta \mathrm{R}<0.2$ around the seed jet barycenter axis.

\subsection{Identification}

Identification of tau leptons is based on variables which provide discrimination between QCD jets and tau leptons. While the charged tracks from the $\tau$ lepton decay are collimated in a narrow cone, tracks from QCD jets tend to spread out (Figure 5).

A projective likelihood (LLH) method, using the log-likelihood-ratio of signal and background, and boosted decision trees (BDT), are used to find the optimal separation in a multidimensional phase space. Figure 6 shows the efficiency as a function of the number of primary vertices and using the LLH method. This demonstrates that the performance of the tau identification algorithms does not degrade as the pile-up increases. To distinguish between electrons and taus one cut-based and one boosted decision tree (BDT)-based veto have been used. Same techniques, as described above for 1-prong taus are used to identify multi-prong taus where additional information based on the tau life-time is used.

\subsection{Identification Efficiency Measurement}

The performance and systematic uncertainties of the tau identification methods are evaluated on data using two different signal channels. The first method use $Z \rightarrow \tau \tau$ events and rely on a tag-and-probe approach which follows closely the $Z \rightarrow \tau \tau$ cross-section measurement [8]. One tau is considered to decay into a muon, which is used to select the event. The second tau lepton decays hadronically and is used to measure the tau identification efficiency. The visible mass of the muon and the hadronic tau is shown in Fig. 7 for data and simulation after applying the tight BDT tau identification and shows a good agreement with the predictions from simulation.

The tau identification efficiency is also studied using $W \rightarrow \tau \nu$ events. The number of hadronic tau candidates are derived from a template fit, which was obtained on the distribution of the number of tracks of the tau. Three different templates are used: true hadronic tau decays, electrons 


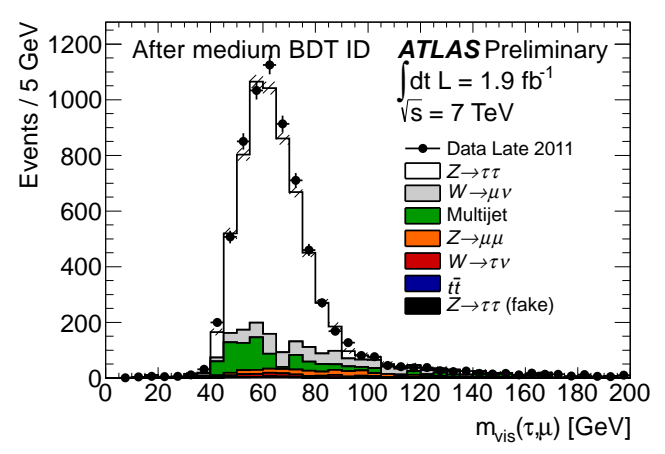

Figure 7: Visible mass of the selected muon and tau candidate for data and simulation after full event selection, but before applying any tau identification. The QCD multi-jet background was obtained by a data-driven method [6].

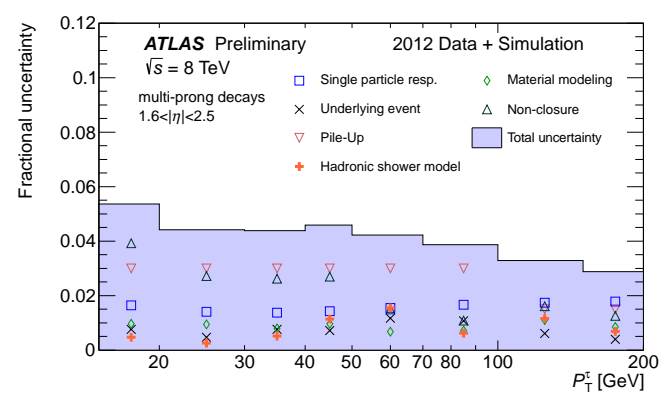

Figure 8: Final systematic uncertainty on the tau energy scale for multi-prong decays in the endcap region, as a function of $p_{T}$. Each different marker represents a separate source of uncertainty as indicated in the legend. The violet band shows the combined uncertainty from all sources [9].

misidentified as tau leptons, and QCD multi-jets misidentified as taus. While the first two are obtained from simulation, the QCD multi-jet template was estimated by a data-driven method. The measured efficiencies in both methods are in good agreement with predictions from simulation within $5 \%(8 \%-12 \%)$ for the $W \rightarrow \tau \nu(Z \rightarrow \tau \tau)$ method.

\subsection{Tau energy scale}

The tau energy scale is restored from the so-called Local Hadron Calibration (LC) used for jets, as tau decays consist of a specific mixture of charged and neutral hadrons [9]. Taking into account the corrections the tau energy can be expressed as

$$
E^{\tau}=\frac{E_{\mathrm{LC}}^{\tau}-E_{\text {pileup }}}{R\left(E_{\mathrm{LC}}^{\tau},\left|\eta_{\text {reco }}^{\tau}\right|, n_{\text {prongs }}\right)}
$$

where $E_{\text {pileup }}$ takes into account the additional energy deposits coming from different collision events and the response $R\left(E_{\mathrm{LC}}^{\tau}, \mid \eta_{\text {reco }}^{\tau}, n_{\text {prongs }}\right)$ is measured by comparing the tau energy at the LC $\left(E_{\mathrm{LC}}^{\tau}\right)$ scale with the true tau visible energy from simulation. This factor also depends on the pseudorapidity $\eta_{\text {reco }}$ of the reconstructed tau and the number of prongs. The uncertainty on the tau energy scale is dominated by the calorimeter response, the pileup correction, the presence of dead material in the calorimeter, the underlying event model and due to deviations of the reconstructed tau kinematics from the true kinematics. The tau energy scale uncertainty as a function of the tau transverse momentum can be seen in Fig 8.

\section{Conclusions}

With the improved algorithms designed for the high pile-up environment, the muon and tau reconstruction and identification have worked very well in 2012. The performance has been checked and no degradation with increase in pile-up has been observed. The results observed in data are well produced by the simulation. 


\section{References}

[1] ATLAS Collaboration, JINST 3, (2008) S08003.

[2] ATLAS Collaboration, ATLAS-CONF-2010-064, https://cdsweb.cern.ch/record/1281339.

[3] ATLAS Collaboration, ATLAS-CONF-2011-008, https://cdsweb.cern.ch/record/1330715.

[4] https://atlas.web.cern.ch/Atlas/GROUPS/PHYSICS/MUON/PublicPlots/2012/June/

[5] ATLAS Collaboration, ATLAS-CONF-2011-046, https://cdsweb.cern.ch/record/1338575.

[6] ATLAS Collaboration, ATLAS-CONF-2011-152, https://cdsweb.cern.ch/record/1398195.

[7] https://twiki.cern.ch/twiki/pub/AtlasPublic/TauPublicCollisionResults

[8] ATLAS Collaboration, Phys.Rev.D 84 (2011) 112006.

[9] ATLAS Collaboration, ATLAS-CONF-2012-054, https://cdsweb.cern.ch/record/1453781 